\title{
A subtle stroke
}

\begin{abstract}
Introduction: The authors present a clinical case of an ischemic stroke that presented with anomic aphasia.

Case report: A 75-year old woman was brought to the emergency department with an anomic aphasia that had started that day. The neurological exam confirmed the anomic aphasia with no other associated findings. The Head-CT Scan showed focal points of ischemic gliosis without any other acute changes that could suggest vascular lesions particularly in the middle cerebral artery territory. The patient was hospitalized in the cerebrovascular disease unit with the diagnosis of ischemic stroke. During her stay at the unit, the patient developed a decreased nasolabial fold prominence on the right side, motor aphasia, dysmetria and a lack of balance while walking. On the fourth day, the patient underwent an MRI that revealed a sub-acute infarction in a partial territory of the left middle cerebral artery with a partially re-canalized thrombus in the inferior M2 branch of this artery. Blood work showed a dyslipidemia. The echocardiogram detected a type 1 diastolic dysfunction with an ejection fraction of 55\%. Patient was discharged after 8 days. Currently, the patient is followed in the cerebrovascular diseases department. The neurological evaluation demonstrates aphasia with some impairment of comprehension and naming, the speech has fluency loss showing occasional anomic pauses and paraphasias.
\end{abstract}

Conclusion: The authors alert to the fact that a stroke can present itself in multiple ways, stressing the role of the clinical symptoms in its diagnosis.
Volume 10 Issue I - 2020

\section{Rafael Ferreira Nascimento, Diogo Raposo André, João Miguel Freitas, João Patrício Freitas, Rafael Freitas}

Department of Internal Medicine, Serviço de Saúde da Região Autónoma da Madeira, Funchal, Portugal

Correspondence: Rafael Ferreira Nascimento, Department of Internal Medicine, Serviço de Saúde da Região Autónoma da Madeira, Funchal, Portugal,Tel +35191215347I,

Email rafaelnasciment@campus.ul.pt

Received: January 28, 2020 | Published: February 12, 2020

\section{Introduction}

When a patient presents himself with changes in verbal expression or understanding the people around him, we usually frame it in the field of the aphasias. Aphasia is frequent in the acute and sub-acute stroke accounting for $1 / 3$ of presentations. ${ }^{1}$ According to studies published to date the frequency of aphasia is located between $21 \%-38 \%{ }^{2-10}$ The remission of aphasia occurs mainly in the first 3 months, but little is known about the recovery course within these 3 months. ${ }^{6,10,11}$ The article shows a case of an anomic aphasia due to an ischemic stroke and the subsequent recovery of the patient.

\section{Case Report}

A 75-year old woman with known hypertension was brought to the emergency department with an anomic aphasia with three and a half hours of evolution. The neurological exam confirmed the anomic aphasia with no other associated findings. Her Head Computed Tomography (Head-CT) Scan showed focal points of ischemic gliosis without any other acute changes, namely density or morphology of brain tissue, that could suggest vascular lesions particularly in the middle cerebral artery territory (Figure 1) showing a National Institutes of Health Stroke Scale (NIHSS) score of 3.

The patient was hospitalized in the cerebrovascular disease unit (CDU) with the diagnosis of ischemic stroke. During her fourth day at the unit, the patient developed a decreased nasolabial fold prominence on the rigth side, motor aphasia, dysmetria and a lack of balance while walking, the patient underwent a Magnetic Ressonance Imaging (MRI) scan that revealed a sub-acute infarction in a partial territory of the left middle cerebral artery with a partially re-canalized thrombus in the inferior M2 branch of this artery and signs of light to moderate ischemic leukoencephalopathy (Figure 2).

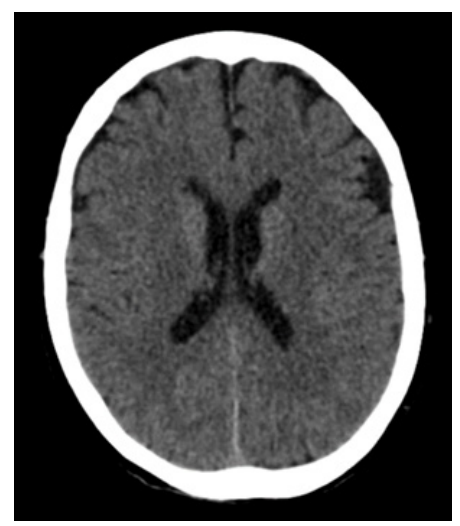

Figure I Head-CT Scan without any acute changes.

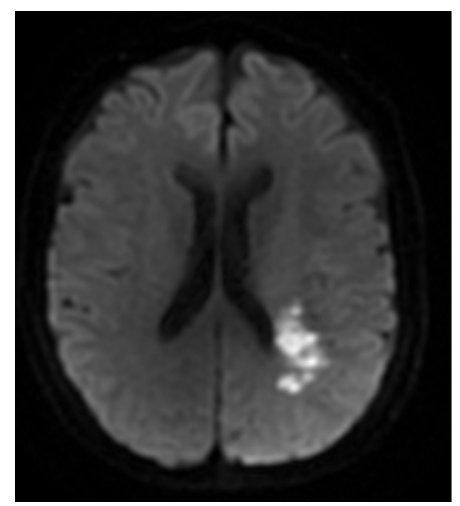

Figure 2 Sub-acute infarction in a partial territory of the left middle cerebra artery with a partially re-canalized thrombus in the inferior $\mathrm{M} 2$ branch. 
Blood work showed a total cholesterol level of $230 \mathrm{mg} / \mathrm{dL}, \mathrm{LDL}$ of $170 \mathrm{mg} / \mathrm{dL}$ and triglycerides of $96 \mathrm{mg} / \mathrm{dL}$ with no other significant alterations. The patient was in sinus rhythm with 80 beats per minute, the transthoracic echocardiogram detected a type 1 diastolic dysfunction with an ejection fraction of $55 \%$. Patient was discharged after 8 days with a NIHSS score of 2 . The eco-doppler to the neck vases shows no significant changes according to age and gender.

Currently, the patient is followed in the cerebrovascular diseases department presenting herself conscious, oriented in space and time without lateralized deficits, although she maintains the aphasia with some impairment of comprehension and nomination. Her speech has fluency loss showing occasional anomic pauses and paraphasias. She also shows a spacial, construtive and ideomotor apraxia associated to an apraxic march with need of supervision on the daily life activities.

\section{Discussion \& conclusion}

The author's presents a clinical case of aphasia due to an ischemic stroke, what is quite peculiar in this case is that the only change at the neurological exam was the presence of an anomic aphasia without any other deficits. Even though the patient had only history of hypertension, this precedent is one of the three most common risk factor for stroke alongside diabetes and atrial fibrillation. ${ }^{12-14}$ Clinical manifestations evolve rapidly during the first hours after the stroke [16] this is particularly true for aphasia. ${ }^{15}$ As we can see during her stay at the CDU the patient developed signs of an ischemic stroke in progression such as decreased nasolabial fold prominence on the right side, motor aphasia, dysmetria and a lack of balance while walking. In this situation in spite of a NIHSS score of 3 , the vigilance was tight in order to take invasive measures if the patient needed so but that was not the case. Studies show that aphasia outcomes remain poor: $32 \%$ to $50 \%$ of aphasics still suffer from aphasia 6 months after stroke. ${ }^{5,6}$ When the patient started being followed in an out-of-thehospital regimen she displayed at first aphasia with some impairment of comprehension and nomination, a speech with fluency loss showing anomic pauses and paraphasias and a spacial, construtive and ideomotor apraxia associated to an apraxic march, taking this into account she started speech and physiotherapy. One month and a half after the discharge from de CDU the speech had become fluent but the patient maintained a low degree of apraxia, showing a balance and gait impairment with an unstable heel to toe walk. Finally, after three months and a half of speech and physiotherapy the patient showed no functional restrictions and no speech impairment, showing a favorable recovery in the time since discharge from the CDU. This article goes to show that the clinical case of an aphasia may be subtle as it was shown but if treated and oriented well, the sequels can be reduced to a minimum.

\section{Acknowledgments}

None.

\section{Conflicts of interest}

The authors declare no conflicts of interest.

\section{References}

1. Hoffmann M, Chen R. The Spectrum of Aphasia Subtypes and Etiology in Subacute Stroke. J Stroke Cerebrovasc Dis. 2013;22(8):1385-1389.

2. Croquelois A, Bogousslavsky J. Stroke aphasia:1500 consecutive cases. Cerebrovasc Dis. 2011;31(4):392-399.

3. Godefroy O, Dubois C, Debachy B, et al. Vascular aphasias: main characteristics of patients hospitalized in acute stroke units. Stroke. 2002;33(3):702-705.

4. Pedersen PM, Jorgensen HS, Nakayama H, et al. Aphasia in acute stroke: incidence, determinants and recovery. Ann Neurol. 1995;38(4):659-666.

5. Pedersen PM, Vinter K, Olsen TS. Aphasia after stroke: type, severity and prognosis. The Copenhagen aphasia study. Cerebrovase Dis. 2004;17(1):35-53.

6. Wade DT, Hewer RL, David RM, et al. Aphasia after stroke: natural history and associated deficits. J Neurol Neurosurg Psychiatry. 1986;49(1):11-16

7. Laska AC, Hellblom A, Murray V, et al. Aphasia in acute stroke and relation to outcome. J Intern Med. 2001;249(5):413-422.

8. Ferro JM, Madureira S. Aphasia type, age and cerebral infarct localization. J Neurol. 1997;244(8):505-509.

9. Hier DB, Yoon WB, Mohr JP, et al. Gender and aphasia in the Stroke Data Bank. Brain Lang. 1994;47(1):155-167.

10. Brust JC, Shafer SQ, Richter RW, et al. Aphasia in acute stroke. Stroke. 1976;7(2):167-174.

11. Lindenstrem E, Boysen G, Christiansen LW, et al. Reliability of Scandinavian Neurological Stroke Scale. Cerebrovasc Dis. 1991;1:103107.

12. Inatomi $\mathrm{Y}$, Yonehara $\mathrm{T}$, Omiya $\mathrm{S}$, et al. Aphasia during the acute phase in ischemic stroke. Cerebrovasc Dis. 2008;25(4):316-323.

13. Engelter ST, Gostynski M, Papa S, et al. Epidemiology of aphasia attributable to first ischemic stroke: Incidence, severity, fluency, etiology, and thrombolysis. Stroke. 2006;37(6):1379-1384.

14. Yamamoto H. Matsumoto M, Hashikawa K, et al. Stroke onset and course. In: Bogousslavsky J, Caplan L, editors. Stroke Syndromes. Cambridge: Cambridge University Press, 2001. pp. 3-7

15. Croquelois A. Wintermark M, Reict M, et al. Aphasia in hyperacute stroike: language follows brain penumbra dynamics. Ann Neurol. 2003;54(3):321-329. 